\title{
Session C
}

\section{Convection in planets and brown}




\title{
Modeling convection and zonal winds in giant planets
}

\author{
Martha Evonuk $^{1}$ and Gary A. Glatzmaier ${ }^{2}$ \\ ${ }^{1}$ Institut für Geophysik, ETH Hoengg, 8093 Zürich, Switzerland \\ email: mevonuk@erdw.ethz.ch \\ ${ }^{2}$ Department of Earth and Planetary Sciences, University of California, Santa Cruz \\ 1156 High Street, Santa Cruz, CA 95064, USA \\ email: glatz@es.ucsc.edu
}

\begin{abstract}
Three basic modeling approaches have been used to numerically simulate fluid turbulence and the banded zonal winds in the interiors and atmospheres of giant planets: shallowwater models, deep-shell Boussinesq models and deep-shell anelastic models. We review these models and discuss the approximations and assumptions upon which they are based. All three can produce banded zonal wind patterns at the surface. However, shallow-water models produce a retrograde (i.e., westward) zonal jet in the equatorial region, whereas strong prograde (i.e., eastward) equatorial jets exist on Jupiter and Saturn. Deep-shell Boussinesq models maintain prograde equatorial jets by the classic method of vortex stretching of convective columnar flows; however, they neglect the effects of the large density stratification in these giant planets. Deepshell anelastic models account for density stratification and maintain prograde equatorial jets by generating vorticity as rising fluid expands and sinking fluid contracts, without the constraint of long thin convective columns.
\end{abstract}

Keywords. Planets and satellites: general, Jupiter, Saturn, stars: interiors

\section{Introduction}

Observations of the latitudinally banded cloud patterns on the surfaces of giant planets like Jupiter (Figure 1) provide rich evidence of turbulent convection in the atmospheres of these planets. The measured motion of the surface clouds also reveals an underlying banded pattern of zonal winds (e.g., Porco et al. 2003). These observations have motivated many theoretical studies to explain the fluid dynamics of the atmospheres of these planets and to predict the dynamics in their deep interiors.

Here we briefly review three approaches to modeling the three dimensional structure of the fluid flow and maintenance of the banded zonal winds. One dimensional evolutionary models predict that convection occurs throughout the interiors of these fluid planets (Guillot 1999). Phase changes certainly affect convection in the shallow atmosphere and likely also play an important role deep within the interior where molecular hydrogen changes to metallic atomic hydrogen; however, here we choose not to cover these issues. Likewise, fluid flows in the semi-conducting outer region and conducting inner region generate electric currents, which maintain the observed magnetic fields; but we will not review this topic here. It is generally believed that the magnetic Lorentz forces are, for the most part, weak compared with the other forces in this problem.

We begin by describing the equations of motion and follow with discussions of the three basic approaches that have been used to solve them. 


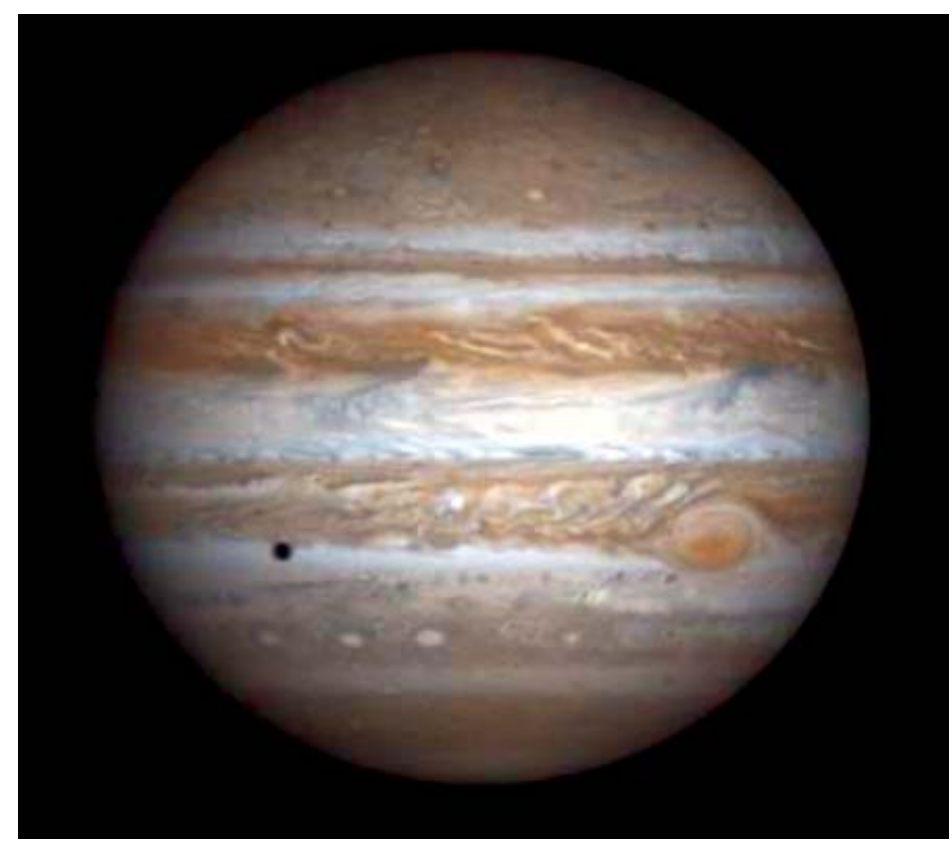

Figure 1. A mosaic of Jupiter taken by Cassini on the 29th of September in 2000 at Cassini's closest approach to Jupiter, which was approximately 10 million kilometers.

\section{Equations and dimensionless parameters}

The equations for modeling the fluid motions in giant planet atmospheres and interiors are conservation of mass (2.1), the momentum equation (2.2), and the heat equation (2.3):

$$
\begin{gathered}
\frac{d \rho}{d t}=-\rho \nabla \cdot \mathbf{u} \\
\rho \frac{d \mathbf{u}}{d t}=\rho \mathbf{g}-2 \rho \boldsymbol{\Omega} \times \mathbf{u}-\nabla P+\rho \nu\left(\nabla^{2} \mathbf{u}+\frac{1}{3} \nabla(\nabla \cdot \mathbf{u})\right) \\
\rho T \frac{d S}{d t}=\nabla \cdot\left(C_{p} \rho \kappa \nabla T\right)+2 \rho \nu\left(e_{i j}^{2}-\frac{1}{3}(\nabla \cdot \mathbf{u})^{2}\right)+\rho Q
\end{gathered}
$$

where $\rho$ is the density, $\mathbf{u}$ the velocity, $\mathbf{g}$ the gravity, $\boldsymbol{\Omega}$ the rotation rate, $P$ the pressure, $\nu$ the kinematic viscosity, $T$ the temperature, $S$ the specific entropy, $C_{p}$ the specific heat capacity, $\kappa$ the thermal diffusivity, $e_{i j}$ the rate of strain tensor, and $Q$ a specified internal heating rate. The viscous term in $(2.2)$ is for a constant dynamic viscosity, $(\rho \nu)$. The equation of state is:

$$
d S=\frac{C_{p}}{T} d T-\frac{\alpha}{\rho} d P
$$

where $\alpha$ is the coefficient of thermal expansion. We will look at various simplifications to these equations and their ramifications in the following sections. 
Some parameters of interest, and their approximate values for Jupiter, are the Ekman number (ratio of viscous to Coriolis forces)

$$
E k=\frac{\nu}{2 \Omega D^{2}} \approx 10^{-18}
$$

and the Rayleigh number (ratio of buoyancy to diffusion)

$$
R a=\frac{g_{o} \alpha \Delta T D^{3}}{\nu \kappa} \approx 10^{24}
$$

where $\mathrm{D}$ is the depth of convection zone, $g_{o}$ the mean background gravity, and $\Delta T$ is the drop in superadiabatic temperature across the convection zone. Jupiter's high Rayleigh number and low Ekman number are far beyond the capabilities of computer simulations and indicate that Jupiter is in a highly turbulent regime. However, an accurate simulation of the large scale dynamics does not require the use of the actual viscous and thermal diffusivities because energy cascades down to small length scales through an inertial range. Instead, enhanced (turbulent) values of these diffusivities suffice as long as enough of the inertial range is captured in the turbulent simulation. However, if the model's diffusivities are too large and the flows are laminar the results are likely unrealistic.

We also note that the density changes greatly through the atmosphere and interior of the giant planets. The number of density scale heights, $N_{\rho}$, where

$$
N_{\rho}=\ln \left(\frac{\rho_{b o t}}{\rho_{t o p}}\right),
$$

from the deep interior to a pressure of $10^{8} \mathrm{~Pa}$ is 5.3 (Guillot 1999), and several more scale heights are present in the atmosphere above $10^{8} \mathrm{~Pa}\left(1 \mathrm{bar}=10^{5} \mathrm{~Pa}\right)$. Shallow-water models completely ignore the deep interior and deep-shell Boussinesq models ignore the density stratification. However, deep-shell anelastic models account for density stratification, which, as we will argue, is the important advantage of this approach.

\section{Modeling approaches}

In this section we discuss three different approaches to modeling the internal fluid dynamics of giant planets. The first is the shallow-water approximation, the second a deep shell assuming the Boussinesq approximation, and the third a deep shell using the anelastic approximation.

\subsection{The shallow-water approximation}

The barotropic approximation, or shallow-water approximation, assumes that the vertical scale of the simulation is small compared to the horizontal scale and that the vertical flow is much smaller than the horizontal flow. Hydrostatic equilibrium is assumed to first order, but the pressure perturbation, horizontal velocities and gravitational acceleration do not vary significantly in the vertical direction. The simplest form of the shallow-water equations (the two-dimensional $f$-plane approximation) is a single layer of inviscid, constant density fluid at latitude, $\lambda$, with variable depth, $h$. The equations for conservation of mass and momentum then reduce to

$$
\begin{gathered}
\frac{\partial h}{\partial t}=-\frac{\partial\left(u_{x} h\right)}{\partial x}-\frac{\partial\left(u_{y} h\right)}{\partial y} \\
\frac{\partial u_{x}}{\partial t}=-u_{x} \frac{\partial u_{x}}{\partial x}-u_{y} \frac{\partial u_{x}}{\partial y}-g_{o} \frac{\partial h}{\partial x}+f u_{y}
\end{gathered}
$$




$$
\frac{\partial u_{y}}{\partial t}=-u_{x} \frac{\partial u_{y}}{\partial x}-u_{y} \frac{\partial u_{y}}{\partial y}-g_{o} \frac{\partial h}{\partial y}-f u_{x}
$$

where the Coriolis parameter is $f=2 \boldsymbol{\Omega} \cdot \hat{\mathbf{z}}=2 \Omega \sin \lambda$ and $\hat{z}$ is the local vertical unit vector. The vorticity of the flow relative to the rotating (Cartesian) frame of reference is $\omega=\nabla \times \mathbf{u}$. Its local vertical component is

$$
\omega_{z}=\frac{\partial u_{y}}{\partial x}-\frac{\partial u_{x}}{\partial y} .
$$

The potential vorticity of the fluid layer at a given horizontal location, $\left(\omega_{z}+f\right) / h$, can be shown to be approximately constant:

$$
\frac{d}{d t}\left(\frac{\omega_{z}+f}{h}\right)=0 .
$$

Therefore the local vertical vorticity increases as fluid horizontally converges and the thickness of the layer increases, and vice versa.

\subsubsection{Rossby waves}

Rossby waves, also known as planetary waves, are low frequency waves with very large length scales and large displacements in latitude. The beta-plane approximation (Verkley 1990; Veronis 1981) allows the Coriolis parameter, $f$, to vary with latitude. It is expanded about a latitude $\lambda_{o}$ such that

$$
f=2 \Omega \sin \lambda \cong 2 \Omega\left(\sin \lambda_{o}+\frac{y}{a} \cos \lambda_{o}+\ldots\right) \cong f_{o}+\beta_{o} y
$$

where $f_{o}$ and $\beta_{o}$ are constants, $a$ is the equatorial radius and $y$ is in the local northward direction. If the local vorticity is small relative to the planetary vorticity, $|\omega|<<\left|f_{o}\right|$, we can assume a wave-like solution and solve for the Rossby dispersion relation,

$$
\omega=-\frac{\beta_{o} R^{2} \ell}{1+R^{2}\left(\ell^{2}+m^{2}\right)}
$$

where $\ell$ and $m$ are the horizontal wave numbers in longitude $(x)$ and latitude $(y)$, respectively, and the radius of deformation is $R=\left(g_{o} h_{\text {mean }}\right)^{1 / 2} / f_{o}$. The zonal (longitudinal) wave speed in the $\mathrm{x}$-direction is $\omega / \ell$, which is negative (i.e., westward) for all $\ell$ and $m$.

Global shallow-water models of fluid turbulence can produce banded zonal winds with jets existing at latitudes with large gradients of potential vorticity. However, the equatorial jet is westward (i.e., retrograde) (e.g., Cho \& Polvani 1996) due to Coriolis forces acting on turbulent eddies. This is also the direction of the zonal flow in the equatorial region of the Earth's atmosphere. However, unlike the Earth, giant planets do not have solid impermeable boundaries at the base of their shallow atmospheres. The observed zonal flows in the equatorial regions of Jupiter and Saturn are eastward (i.e., prograde), which strongly suggests that the dynamics of the deep interiors of these planets are important and cannot be neglected. This also indicates that the basic mechanism maintaining differential rotation in giant planets is not solely operating in latitude and longitude; that is, the dynamics in the radial dimension are critical and cannot be averaged out as done in shallow-water models.

Resolution in radius has been achieved with multiple layer models, such as EPIC (Dowling et al. 1998), which act like global circulation models for planetary atmospheres. However, because of their hydrostatic balance and subadiabatic requirements, they cannot simulate thermal convection. Usually, latitudinal differential rotation is forced in these models by prescribing it at the lower boundary. That is, it is assumed that differential rotation is maintained by deep thermal convection below the shallow atmosphere 
and possibly modified to some extent within the atmosphere. Alternatively, some 2D barotropic models obtain a latitudinally banded zonal wind structure by simply forcing it at the upper boundary (Showman et al. 2006) to see how it decays with depth.

\subsection{Deep-shell Boussinesq simulations}

Deep-shell Boussinesq simulations have been used by many authors (e.g., Christensen 2002; Heimpel et al. 2005) to study the dynamics of giant-planet interiors. In this section we discuss the Boussinesq approximation and the vortex stretching of geostrophic columnar convection that self-consistently maintains differential rotation with an eastward equatorial jet - without prescribing a latitudinal forcing at some depth.

\subsubsection{Boussinesq approximation}

The Boussinesq approximation for thermal convection (Boussinesq 1903) neglects the variation of density except in the buoyancy term after subtracting out hydrostatic equilibrium $\left(\nabla P_{o}=\rho_{o} \mathbf{g}\right)$. It assumes the divergence of velocity vanishes, so

$$
\nabla \cdot \mathbf{u}=0 .
$$

The Boussinesq approximation is most relevant for liquids that are nearly incompressible with flow speeds small relative to the sound speed. Equation (3.2) ensures conservation of mass and fluid volume. The elimination of sound waves allows much larger numerical time steps, which are constrained by a CFL condition based on the fluid velocity instead of the much larger sound speed. For an extended discussion of the Boussinesq equations and their simplifying assumptions see Spiegel \& Veronis (1960) and Mihaljan (1962).

Density perturbations due to pressure perturbations are neglected in the Boussinesq approximation and the local perturbations in the density are assumed to be small compared to the constant background density $\left(\rho_{o}\right)$, as is the total drop in density across the convection zone. The density perturbation $\rho^{\prime}$ appears only in the buoyancy term and depends only on the temperature perturbation, $T^{\prime}=T-T_{o}$ :

$$
\rho=\rho_{o}+\rho^{\prime}=\rho_{o}\left(1-\alpha\left(T-T_{o}\right)\right)
$$

where $T_{o}$ is the constant background temperature. With the neglect of pressure perturbations in (2.4), entropy perturbations are proportional to temperature perturbations:

$$
T_{o} d S=C_{p} d T
$$

The background state is therefore both isothermal and adiabatic.

The diffusivities are usually assumed to be constants and viscous heating is neglected, so the Boussinesq versions of the momentum and heat equations are:

$$
\begin{gathered}
\frac{\partial \mathbf{u}}{\partial t}=-(\mathbf{u} \cdot \nabla) \mathbf{u}-\alpha T^{\prime} \mathbf{g}-2 \boldsymbol{\Omega} \times \mathbf{u}-\frac{1}{\rho_{o}} \nabla P^{\prime}+\nu \nabla^{2} \mathbf{u}, \\
\frac{\partial T^{\prime}}{\partial t}=-(\mathbf{u} \cdot \nabla) T^{\prime}+\kappa \nabla^{2} T^{\prime}+\frac{Q}{C_{p}}
\end{gathered}
$$

\subsubsection{The Taylor-Proudman theorem}

The geostrophic approximation is made when, after subtracting out hydrostatic equilibrium, the pressure gradient and Coriolis forces have much greater amplitudes than the other forces in the momentum equation:

$$
\nabla P=2 \rho_{o} \mathbf{u} \times \mathbf{\Omega} .
$$




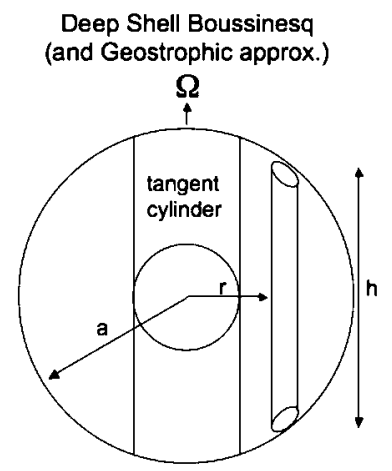

a) Meridional Plane

(convective column)

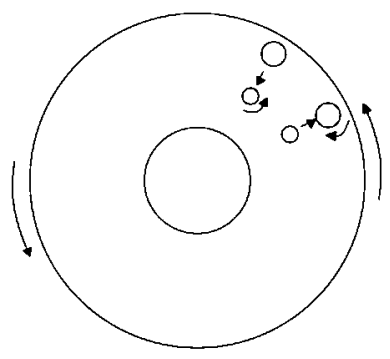

b) Equatorial Plane

(expansion and contraction determined

by spherical boundaries)

Figure 2. Schematic of convective columns and vorticity generation in the deep-shell Boussinesq approximation. The meridional plane (a) shows a convective column outside the tangent cylinder. As the column moves away from the axis of rotation the boundaries force the column's height to decrease and its diameter to increase leading to the generation of negative vorticity. This can also be seen in cross-section in the equatorial plane (b).

The curl of the above equation gives the Taylor-Proudman theorem:

$$
\frac{\partial \mathbf{u}}{\partial z}=0
$$

where now $z$ is the cylindrical coordinate parallel to the constant planetary rotation vector, $\boldsymbol{\Omega}=\Omega \hat{\mathbf{z}}$. That is, to first order velocity does not change in the direction parallel to $\boldsymbol{\Omega}$. Because of the impermeable outer boundary, velocity parallel to $\boldsymbol{\Omega}$ is small relative to that perpendicular to $\boldsymbol{\Omega}$. Consequently, the geostrophic approximation results in convective columns with axes parallel to the planetary rotation axis that span the convection zone (Figure 2). However, the geostrophic approximation is not necessarily valid for turbulent interiors of giant planets where nonlinear advection is important.

\subsubsection{Vorticity generation due to vortex stretching}

Nonlinear advection is included in a potential vorticity theorem for convective columns. If $h$ in (3.1) is now defined as the height of a convective column (parallel to the planetary rotation axis) and $f=2 \Omega$ is the total planetary vorticity, the potential vorticity of these constant density convective columns can be shown to be approximately conserved. However, instead of vorticity decreasing with latitude as in the shallow-water models, it now increases as fluid moves moves toward the rotation axis (when the column is 
outside the cylinder tangent to the equator of the inner boundary, Figure 2a). That is, for an inviscid, rotating, spherical fluid shell, as fluid in a column moves away from the planetary rotation axis (i.e., rises) the height of the fluid column is forced to decrease by the spherical boundaries, which forces the diameter of the column to increase. This in turn results in Coriolis forces that generate negative vorticity (Figure 2b). The opposite occurs for sinking fluid. This process causes the pattern (phase) to propagate eastward in longitude (i.e., prograde). In addition, due to the curvature of the spherical boundary, this effect increases with distance from the rotation axis and ultimately causes rising fluid to tilt eastward and sinking fluid to tilt westward. The resulting nonlinear convergence of eastward momentum flux in the outer part of the convective column and convergence of westward momentum flux in the inner part of the column maintains a differential rotation (Busse 1976).

While the deep-shell Boussinesq model can maintain a banded surface zonal flow pattern with a prograde equatorial jet similar to those seen on Jupiter and Saturn, its primary weaknesses are its neglect of density stratification effects and its reliance on the geostrophic approximation (laminar convective columns spanning the convective zone) to generate vorticity. However, the deep interiors of giant planets are strongly turbulent and density stratified. Also, there would be problems if a much smaller (i.e., more realistic) viscosity were used in a Boussinesq simulation. Correspondingly smaller convective driving would be needed to maintain columnar flow structure, which is required to maintain the differential rotation. The resulting convective columns would be thinner by orders of magnitude; and this would produce zonal jets with latitudinal extents orders of magnitude smaller than those observed on our giant planets (Glatzmaier et al. in prep.).

\subsection{Deep-shell anelastic simulations}

The internal flows in giant planets have been studied with $2 \mathrm{D}$ and $3 \mathrm{D}$ simulations of rotating convection in a deep density-stratified fluid using the anelastic approximation (Evonuk \& Glatzmaier 2006a,b; Glatzmaier et al. in prep.). These simulations produce differential rotation without relying on cohesive convective columns confined within impermeable spherical boundaries. Instead, vorticity is generated locally due to the expansion and contraction of turbulent fluid parcels as they move through the density stratification.

\subsubsection{Anelastic approximation}

The anelastic approximation (Gough 1969; Glatzmaier \& Gilman 1981a; Lipps 1990), like the Boussinesq approximation, filters out sound waves and thermodynamic perturbations need to be small relative to an adiabatic hydrostatic background state. However, unlike the Boussinesq approximation, the background state is not at a constant temperature and density. Instead the background state can have realistic stratifications of density and temperature and these strongly influence the dynamics.

For the anelastic approximation $\nabla \cdot(\bar{\rho} \mathbf{u})=0$, where variables with overbars represent background functions that vary with radius. The divergence of velocity is therefore

$$
\nabla \cdot \mathbf{u}=-\frac{u_{r}}{\bar{\rho}} \frac{d \bar{\rho}}{d r}
$$

The time rate of change of density does not vanish, but is small relative to the three parts of the mass flux divergence. The time dependent density perturbation is computed from the pressure and the entropy perturbations,

$$
\rho^{\prime}=\left(\overline{\frac{\partial \rho}{\partial S}}\right)_{P} S^{\prime}+\left(\overline{\frac{\partial \rho}{\partial P}}\right)_{S} P^{\prime} .
$$


Again, the background state satisfies hydrostatic equilibrium, so the anelastic momentum equation is

$$
\bar{\rho} \frac{\partial \mathbf{u}}{\partial t}=-\bar{\rho}(\mathbf{u} \cdot \nabla) \mathbf{u}-\rho^{\prime} \overline{\mathbf{g}}-2 \bar{\rho} \boldsymbol{\Omega} \times \mathbf{u}-\nabla P^{\prime}+\nabla \cdot\left[2 \bar{\rho} \bar{\nu}\left(e_{i j}-\frac{1}{3}(\nabla \cdot \mathbf{u}) \delta_{i j}\right)\right] .
$$

The simplest version of the heat equation for the entropy perturbation $\left(S^{\prime}\right)$ relative to the adiabatic background state is

$$
\bar{\rho} \bar{T} \frac{\partial S^{\prime}}{\partial t}=-\bar{\rho} \bar{T}(\mathbf{u} \cdot \nabla) S^{\prime}+\nabla \cdot\left(\bar{T} \bar{\rho} \bar{\kappa} \nabla S^{\prime}\right)+2 \bar{\rho} \bar{\nu}\left(e_{i j}^{2}-\frac{1}{3}(\nabla \cdot \mathbf{u})^{2}\right)+\bar{\rho} Q .
$$

Typically though, a convergence of radiative and/or conductive heat flux driven by a temperature gradient is also included.

\subsubsection{Rhines' scaling}

Rhines' scaling (Rhines 1975) represents an inverse energy cascade transferring energy to small frequencies and large length scales culminating at a characteristic length scale, the "Rhines' scale" $\left(\frac{2 U}{\beta}\right)^{1 / 2}$, for which the effects of advection $(U)$ become comparable to those of Coriolis forces $(\beta)$. Assuming the geostrophic approximation, the surface expression of a Rhines' scaling on a sphere can be calculated for both the Boussinesq case and the anelastic case. As described above, $\beta$ is

$$
\beta(\lambda)=\frac{2 \Omega \cos \lambda}{a},
$$

the latitudinal derivative of the Coriolis parameter, where again $\lambda$ is the latitude and $a$ is the planetary radius. This latitudinal profile can be compared with $d^{2} \bar{u} / d y^{2}$ north and south of the equatorial jet of a giant planet, as in Ingersoll \& Pollard (1982). A variation on the $\beta$ scale has also been used by Heimpel, Aurnou \& Wicht (2005) to compare their Boussinesq columnar convection simulations to Jupiter. The equivalent of $\beta$ for anelastic columnar convection is $B \sin ^{2} \lambda$ (Ingersoll \& Pollard 1982) where

$$
B(r)=\frac{2 \Omega}{M} \frac{d M}{d r} .
$$

Here $\mathrm{r}$ is the cylindrical radius and $M=\int_{-h / 2}^{+h / 2} \rho d z$. Ingersoll \& Pollard (1982) find that $B \sin ^{2} \lambda$ is more in agreement with Jupiter and Saturn profiles than $\beta$, implying that a deep anelastic fluid shell is a better representation of the interior of a giant planet than a Boussinesq shell. However, as mentioned, columnar convection is not necessary or even likely in the turbulent interiors of these planets. Next we examine the role of potential vorticity in an anelastic fluid without the constraint of columnar flow.

\subsubsection{Vorticity generation due to density stratification}

If buoyancy, viscosity and vortex stretching are dropped, the curl of the anelastic momentum equation reduces to the following conservation of potential vorticity:

$$
\frac{d}{d t}\left(\frac{2 \Omega+\omega_{z}}{\bar{\rho}}\right)=0
$$

where $\omega_{z}$ is vorticity parallel to and relative to the planetary rotation rate $\Omega$. That is, the expansion velocity of a rising fluid parcel produces Coriolis forces that decrease $\omega_{z}$ (i.e., generate negative vorticity relative to the rotating frame); and the opposite occurs for sinking fluid (Glatzmaier \& Gilman 1981b). There is no requirement for laminar convective columns spanning the fluid from the northern to southern spherical boundaries 

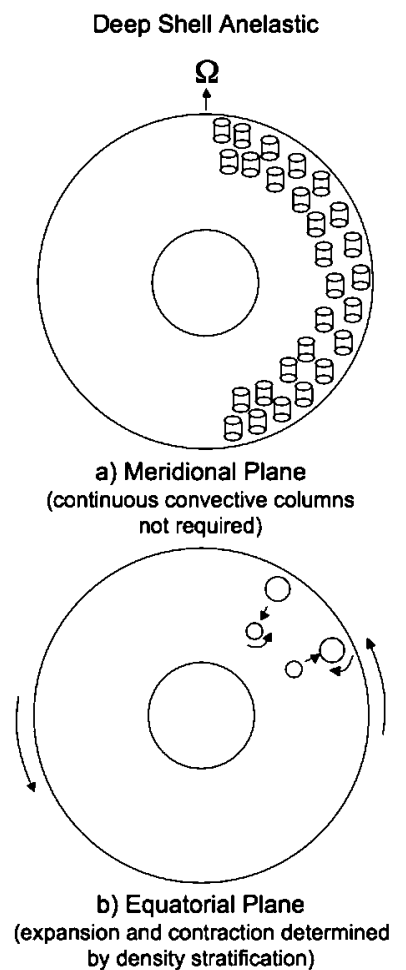

Figure 3. Schematic of convective columns in a deep anelastic shell. Multiple disconnected axially-aligned vortices fill the interior (a). Their radial movement through the smaller density scale height near the outer boundary causes the convergence of eastward momentum in the outer part of the equatorial region (b).

(Figure 3a). Instead, turbulent fluid parcels and isolated vortices experience this potential vorticity conservation. In addition, the smaller density scale height near the outer boundary in giant planets cause rising and sinking fluid to tilt in longitude in a way that converges eastward momentum in the outer part of the equatorial region (Figure $3 \mathrm{~b}$ ), producing a prograde equatorial jet there (Glatzmaier et al. in prep.).

\section{Conclusions}

All three types of models produce banded zonal flows at the surface. However, only the deep models naturally maintain an eastward equatorial jet as observed on the gas giants Jupiter and Saturn. As computer simulations resolve more turbulence (i.e., approach reality) the long thin convective columns required in Boussinesq models become less likely and the local density-stratification mechanism becomes increasingly important for maintaining differential rotation and eastward equatorial flow.

\section{Acknowledgements}

Support for this research has been provided by the NASA Outer Planets Research program (NNG05GG69G). Computational resources were provided by NASA (Ames Research Center), NSF (Pittsburgh Supercomputing Center) and DOE (NERSC). 


\section{References}

Boussinesq, J. 1903, Theorie analytique de la chaleur (Paris: Gauthier-Vellars), 2, 172

Busse, F.H. 1976, Icarus 29, 255

Cho, J. Y-K. \& Polvani, L.M. 1996, Phys. Fluids 8, 1531

Christensen, U.R. 2002, J. Fluid Mech. 470, 115

Dowling, T.E., Fischer, A.S., Gierasch, P.J., Harrington, J., LeBeau, R.P. \& Santori, C.M. 1998, Icarus 132,221

Evonuk, M. \& Glatzmaier, G.A. 2006a, Icarus 181, 458

Evonuk, M. \& Glatzmaier, G.A. 2006b, Planet. and Space Sci. in press

Glatzmaier, G.A., Evonuk, M. \& Rogers, T.M. in prep.

Glatzmaier, G.A. \& Gilman, P.A. 1981a, ApJS 45, 335

Glatzmaier, G.A. \& Gilman, P.A. 1981b, ApJS 45, 381

Gough, D.O. 1969, J. Atmos. Sci. 26, 448

Guillot, T. 1999, Science 286, 72

Heimpel, M., Aurnou, J. \& Wicht, J. 2005, Nature 438, 193

Ingersoll, A.P. \& Pollard, D. 1982, Icarus 52, 60

Lipps, F.B. 1990, J. Atmos. Sci. 47, 1794

Mihaljan, J.M. 1962, ApJ 136, 1126

Porco, C.C., et al. 2003, Science 299, 1541

Rhines, P.B. 1975, J. Fluid Mech. 69, 417

Showman, A.P., Gierasch, P.J. \& Lian, Y. 2006, Icarus 182, 513

Spiegel, E.A. \& Veronis, G. 1960, ApJ 131, 442

Verkley, W.T.M. 1990, J. Atmos. Sci. 47, 2453

Veronis, G. 1981, Evolution of Physical Oceanography. Massachusetts Institute of Technology Press, 140

\section{Discussion}

ANDREW INGERSOLL: What lower boundary condition did you assume in your Jupiter model? Did you take the magnetic field and electrical conductivity into account?

GLATZMAIER: My anelastic 3D global simulations of convection and magnetic field generation in the interior of a giant planet has been running for several years now. The MHD equations are solved simultaneously with the anelastic equations of motion, with full nonlinear Lorentz forces and Ohmic heating. I've tested both stress-free and non-slip lower boundary conditions on the velocity. The non-slip conditions seem to produce flows in better agreement with the surface observations of Jupiter and Saturn - maybe because there would be magnetic torques between the upper convection zone and the lower, much more slowly flowing and more highly conducting interior. The electrical conductivity in the model increases by about a factor of 1000 with depth through the semi-conducting region, as it is believed to do in Jupiter and Saturn. Convective flows are largest near the surface, where of course the density is smallest. Consequently, there is a fairly shallow region within the semi-conducting region where the conductivity is high enough and the fluid flows are strong enough to generate magnetic fields.

HANS-G. Ludwig: How do your models of giant planets relate to the work of Cho and collaborators who obtain the banded cloud structure of the solar system giant planets from $2 \mathrm{D}$ shallow-water like models?

Glatzmaier: The shallow water model results produce banded zonal winds, but with a large retrograde jet in the equatorial region, opposite of what is observed on Jupiter and Saturn. However, by prescribing a thermocline as a function of latitude in a shallow water model you can drive a thermal wind that fits the observations or any profile you 
like. But by doing so you assume the zonal wind is only a thermal wind, i.e., driven by Coriolis forces resulting from meridional circulation that is driven by your highly-tuned artificially-prescribed latitudinally-dependent bottom heating. We do not prescribe anything as a function of latitude in our 3D model; and we self-consistently get a strong prograde equatorial jet with bands of alternating zonal winds at higher latitude. Meridional circulation in our simulations is weak and so does not drive the zonal winds; the zonal winds are driven by the nonlinear convergence of angular momentum flux, which is determined by the structure of the deep $3 \mathrm{D}$ convection.

I.W. Roxburgh: Could you give some details of what went into the model of solar convection and gravity waves?

Glatzmaier: Tamara Rogers and I developed a 2D anelastic model to study the dynamics in the equatorial plane of the sun. The outer region is superadiabatic and so drives turbulent convection. The inner region, to the center, is strongly subadiabatic, like the solar radiative region, and therefore supports internal gravity waves. These waves are excited by downwelling convective plumes, which are continuously pounding on the stable region from above at all longitudes. We analyze the resulting kinetic energy spectrum and dispersion relation of the gravity waves as a function of depth below the convection zone. In addition we monitor the transport and deposition of angular momentum by the gravity waves in the stable region to study how they may be affecting the rotation profile of that region. We are currently incorporating these features in my $3 \mathrm{D}$ global (solar) dynamo model. 